\title{
Drug Prescribing Trends among Consultants and General Practitioners in Sharjah-UAE
}

\author{
Suleiman I. Sharif*, Hoda Fazli, Yasamin Tajrobehkar, Zeinab Namvar, \\ Laila M. T. Bugaighis \\ Department of Pharmacy Practice \& Pharmacotherapeutics, College of Pharmacy, University of Sharjah, \\ Sharjah, United Arab Emirates \\ Email: ${ }^{*}$ sharifsi@sharjah.ac.ae
}

Received 28 July 2015; accepted 21 August 2015; published 24 August 2015

Copyright (C) 2015 by authors and Scientific Research Publishing Inc.

This work is licensed under the Creative Commons Attribution International License (CC BY). http://creativecommons.org/licenses/by/4.0/

(c) (7) Open Access

\section{Abstract}

Background: Inappropriate prescribing can lead to errors in dispensing medications and serious problems for patients. Objectives: Prescription analysis can identify such drawbacks of prescribing, increase awareness of prescribers of rational prescribing and consequently lead to proper delivery of pharmaceutical care and enhance therapeutic outcomes. Methods: In the present study, prescriptions issued by consultants from a hospital and by general practitioners from private practice in Sharjah-United Arab Emirates were analyzed using indicators suggested by World Health Organizations. These include information with regard to prescriber, patient and the medication prescribed. We also determined the average number of drugs/encounter and \% of prescriptions with antibiotics and those with injections. Data were collected and analyzed using Microsoft Excel ${ }^{\circledR}$ and expressed in terms of both counts and percentages. Results: Almost all prescriptions were handwritten with easily readable ones being $65 \%$ for consultants and $46 \%$ for general practitioners. Average number of drugs/encounter was 2.1 and 2.8 for consultants and general practitioners, respectively. Antibiotics were prescribed in $27 \%$ and $44 \%$; generic prescribing was $5 \%$ and $10 \%$ by consultants and general practitioners respectively and $8 \%$ of prescriptions by consultants contained injections. Variable results were obtained on information regarding the patient but consultants seem to be better in documenting patient's age and gender. Consultants and general practitioners tend to prescribe 3 drugs and more in $35 \%$ and $25 \%$ respectively. The most commonly prescribed therapeutic classes for both groups of prescribers were NSAIDs and antibiotics with ibuprofen and amoxicillin-clavulanic acid combination being the most commonly prescribed drugs of each class. Conclusion: To improve prescription writing, interventions must include, among others, incorporation of topics on prescription writing in medical curriculum and programs of continuing medical education.

*Corresponding author.

How to cite this paper: Sharif, S.I., Fazli, H., Tajrobehkar, Y., Namvar, Z. and Bugaighis, L.M.T. (2015) Drug Prescribing Trends among Consultants and General Practitioners in Sharjah-UAE. Pharmacology \& Pharmacy, 6, 374-379. 


\section{Keywords}

\section{Prescription Analysis, Trends, Consultants, General Practitioners}

\section{Introduction}

Inappropriate use of medications in healthcare facilities is a common problem not only in developing but also in developed countries [1]-[4]. However, the situation is worse in developing countries as in addition to the deleterious effects on health care outcomes; it also exhausts the limited health budget. In the latter countries, the excessive and indiscriminate use of drugs significantly and advertently influences health care delivery as it increases the incidence and seriousness of adverse drug reactions and interactions and bacterial resistance [1]. The physician has to prescribe medicines that meet the patient clinical need at doses adequate to cover their individual requirements for adequate period of time and at affordable cost [5]. Dispensing errors can be dangerous [6]-[8]. This entails the dispenser to provide the patient with the appropriate strengths of the prescribed drugs and clearly indicating to the patient the appropriate dose and how to measure, administer and frequently use the medicine. Changing a medication to ensure affordability requires a consultation of the prescriber. The dispenser, the pharmacist, must also provide some instruction on proper storage of medicines. The role of the consumer requires strict adherence to the instruction given by both the prescriber and the dispenser to avoid over dosage, under treatment, drug interactions and degradation of active constituents of medicines due to bad storage conditions. Among the easiest ways to examine an aspect of drug misuse is prescription analysis. Such investigation examines the patterns of drug prescribing. Moreover, prescription analysis whether of private practice or that of health facilities can determine areas for improvement towards rational drug use. Moreover, feeding back results of prescription analysis to the actual prescribers and health authorities is a useful method of intervention [1]. In the present study, prescribing behavior, dispensing and use of medicines will be approached to pinpoint various factors that may influence rational drug use.

\section{Methods}

For studying the prescribing trends among consultants in a general hospital, and general practitioners, a total 1239 and 980 prescriptions were collected during the month of April, 2011 from a general hospital out-patients pharmacy and a community pharmacy in Emirate of Sharjah respectively. The prescriptions from the hospital were issued by consultants while those of the pharmacy represent general practitioners (GPs). Prescriptions were subjected to analysis using the World Health Organization (WHO) suggested indicators [9]. These include prescriber's information such as name, registration number and signature; patient's information including the name, age, sex, and address of patient plus brief diagnosis and history of allergy. All prescriptions were examined for eligibility. In addition information on the medicine were determined including dosage regimen, number of encounters per prescription, \% generic drugs prescribed, most common therapeutic classes and the most common drug of each class. Prescriptions were also examined for medications prescribed as injections. In this study, names of patients were concealed in consideration of patients' privacy protection. Data were collected and analyzed using Microsoft Excel ${ }^{\circledR}$ and expressed in terms of both counts and percentages.

\section{Results}

In the present study, most prescriptions were hand written with 35\% - 50\% of them being difficult to read making dispensing at increased risk of errors. All prescriptions issued by consultants or general practitioners (GPs) were deficient in important information regarding the prescriber, the patient and the dosage regimen (Table 1 and Table 2). The average number of drugs per prescription was 2.1 for consultants and 2.8 for general practitioners. Prescribing generic drugs was higher in the case of GPs (10\%) as compared to consultants (5\%). The trend for poly-pharmacy was more evident in prescriptions by consultants where prescriptions with 4 or more drugs totaled to $16 \%$ (Table 3). Prescriptions by consultants contained injections (8\%) as compared to none of those issued by GPs. As shown in Table 1, prescribing two drugs of the same therapeutic class was more in case of GPs as it counted to $16 \%$ of all prescriptions while it was evident in only $3 \%$ of prescriptions by consultants. 


\section{S. I. Sharif et al.}

Table 1. Comparison of \% presence of prescriber's information, eligibility of prescription, average number of drugs/encounter, injections, antibiotics and medication information in prescriptions by consultants and general practitioners.

\begin{tabular}{|c|c|c|}
\hline \multirow{2}{*}{ Information } & \multicolumn{2}{|c|}{ Number (\%) of prescriptions } \\
\hline & Consultants $(\mathrm{n}=1239)$ & General practitioners $(\mathrm{n}=980)$ \\
\hline \multicolumn{3}{|l|}{ Prescriber’s } \\
\hline Name & $1090(88 \%)$ & $813(83 \%)$ \\
\hline Signature & 1115 (90\%) & $941(96 \%)$ \\
\hline Specialty & $991(80 \%)$ & 725 (74\%) \\
\hline License's number & $558(45 \%)$ & 333 (34\%) \\
\hline \multicolumn{3}{|l|}{ Prescriptions } \\
\hline Typed & $0(0 \%)$ & $59(6 \%)$ \\
\hline Hand written & $1090(100 \%)$ & $921(94 \%)$ \\
\hline Easily readable & 805 (65\%) & $451(46 \%)$ \\
\hline \multicolumn{3}{|l|}{ Medication } \\
\hline Average number of drugs/encounter & 2.1 & 2.8 \\
\hline Dose & $620(50 \%)$ & 892 (91\%) \\
\hline Route of administration & $372(30 \%)$ & $186(19 \%)$ \\
\hline Duration of treatment & $942(76 \%)$ & $608(62 \%)$ \\
\hline Generic drugs & $62(5 \%)$ & $98(10 \%)$ \\
\hline Injections & $37(3 \%)$ & $0(0 \%)$ \\
\hline Antibiotics & 335 (27\%) & $431(44 \%)$ \\
\hline Two drugs of the same class & $37(3 \%)$ & $431(44 \%)$ \\
\hline Possible drug-drug interaction & $50(4 \%)$ & $44(4.5 \%)$ \\
\hline
\end{tabular}

Table 2. Comparison of patient's information present in prescriptions by consultants and general practitioners.

\begin{tabular}{ccc}
\hline \multirow{2}{*}{ Patient's information } & \multicolumn{2}{c}{ Number (\%) of prescriptions } \\
\cline { 2 - 3 } Name & Consultants $(\mathrm{n}=1239)$ & General practitioners $(\mathrm{n}=980)$ \\
Age & $1202(97 \%)$ & $941(96 \%)$ \\
Gender & $1115(90 \%)$ & $294(30 \%)$ \\
Address & $1090(88 \%)$ & $353(36 \%)$ \\
Brief diagnosis & $0(0 \%)$ & $137(14 \%)$ \\
History of allergy & $0(0 \%)$ & $7(0.7 \%)$ \\
\hline
\end{tabular}

Table 3. Number and \% of prescriptions with various numbers of medications issued by consultants and general practitioners.

\begin{tabular}{ccc}
\hline \multirow{2}{*}{ Number of drugs } & \multicolumn{2}{c}{ Number (\%) of prescriptions } \\
\cline { 2 - 3 } No drugs & Consultants (n = 1239) & General practitioners (n = 980) \\
One drugs & $25(2 \%)$ & $0 \%)$ \\
Two drugs & $434(35 \%)$ & $470(48 \%)$ \\
Three drugs & $319(28 \%)$ & $265(27 \%)$ \\
Four drugs & $235(19 \%)$ & $157(16 \%)$ \\
More than 4 drugs & $99(8 \%)$ & $59(6 \%)$ \\
\end{tabular}


Drug interactions were observed in $4 \%$ and $4.5 \%$ of encounters by consultants and GPs respectively (Table 1) counted to The most commonly prescribed class of drugs was the NSAIDs constituting $30 \%$ and $51 \%$ for consultants and general practitioners respectively (Table 4). In both cases ibuprofen was the most commonly prescribed of this class as more than $50 \%$ of prescriptions contained this drug. Antibiotics come second on the list. These drugs were prescribed in $27 \%$ and $44 \%$ of prescriptions by consultants and GPs respectively. The combination of amoxicillin and clavulanic acid was the favorite drug. The third class of drugs prescribed by consultants was gastrointestinal drugs (22\%) and vitamins (36\%) for general practitioners (Table 4).

\section{Discussion}

The present study aimed at comparing prescribing behavior of consultants in a hospital and GPs in private clinics. Prescription analysis can indicate areas of irrational drug use practice. Name of patient was deficient in very small \% of prescriptions by both consultants and GPs. These results are similar to those reported for Saudi Arabia [2] [10]. Age of patient was not mentioned in $70 \%$ of prescriptions by GPs as compared to $10 \%$ of encounters by consultants. In the present study, lack of sex and age from prescriptions by consultants was within the ranges mentioned in the above Saudi studies.

Gender of patients was not mentioned in $64 \%$ of prescriptions by GPs as compared to $12 \%$ of those by consultants. Such information are essential for pharmacists dispensing these prescriptions to check on whether doses prescribed were appropriate or not and also in case of female patients to take precautions in cases of pregnancy and lactation. Similar to previous observations in Saudi Arabia [2], patient's address was present in only $14 \%$ of prescriptions by GPs and not mentioned at all in those by consultants. This is alarming since both the prescribing physician and the dispensing pharmacist need to immediately contact the patient in case of either prescription or dispensing error. In the present study diagnosis and history of allergy were completely omitted from all studied prescriptions. This is in contrast to results reported by others in Saudi Arabia [2] [10] [11] but similar to our previous observations in Sharjah [12].

In the present study, the name, signature, specialty and license number of the prescriber were not mentioned in small \% of prescriptions. The percentage lack of such information was rather similar with both groups of prescribers. Most prescriptions analyzed were hand written with readability being of $65 \%$ and $46 \%$ for consultants and GPs respectively. Our results are less than those reported for Saudi Arabia [2] but far in excess of those reported in other studies [3] [10]. Poor legibility of handwriting can lead to misinterpretation by the pharmacist and result in errors in drug dispensing and administration [13] with consequent risks to the patient.

The average number of drugs per prescription for consultants was only slightly higher than that recommended by WHO [9]. However, in prescriptions by GPs the number of drugs/encounter was 2.8 which is similar to that described in India [14] [15]. In the present study, the \% of encounters with more than 4 drugs for both consultants (8\%) and GPS (6\%) was markedly less than that observed in encounters by consultants (25\%) in our earlier study in Sharjah [12].

Surprisingly, the dose of the drug was not mentioned in only 9\% of GPs prescriptions as compared to 50\% of prescriptions by consultants. These results on GPs patterns of prescribing seem, to some extent, better than those

Table 4. Most commonly prescribed therapeutic classes and drug of each class in prescriptions by consultants and general practitioners.

\begin{tabular}{cccc}
\hline \multicolumn{4}{c}{ The most commonly prescribed therapeutic class and drug of each class } \\
Number $(\%)$ of prescriptions & \\
\hline Consultants $(\mathrm{n}=1239)$ & & General practitioners $(\mathrm{n}=980)$ \\
\hline NSAIDs & $372(30 \%)$ & NSAIDs & $500(51 \%)$ \\
Ibuprofen & $644(52 \%)$ & Ibuprofen & $666(68 \%)$ \\
Antibiotics & $335(27 \%)$ & Antibiotics & $431(44 \%)$ \\
Amoxicillin + clavulanic acid & $818(66 \%)$ & Amoxicillin + clavulanic acid & $843(86 \%)$ \\
GI drugs & $273(22 \%)$ & Vitamins & $353(36 \%)$ \\
\hline
\end{tabular}


of consultants. Reasons for such negligence could be attributed to overconfidence of consultants, ignorance, short consultation time or stressful conditions of work. None of these can be an accepted justification for such behavior. Route of administrations was lacking in about $70 \%$ and $80 \%$ of prescriptions by consultants and GPs respectively. On the other hand, duration of treatment was lacking in $24 \%$ and $38 \%$ of prescriptions by consultants and GPs respectively. Such prescribing trends of both consultants and GPs although it is not appropriate; it strongly emphasizes the role of pharmacists in providing rational pharmaceutical care through complementing the prescriber's deficiencies, if any, in dispensed prescriptions.

Generic prescribing was low in prescriptions by both groups of prescribers and even lower than that reported in our earlier study in Sharjah [12] and in some Western countries [3]-[5]. This is may be attributable to a more influential role of pharmaceutical promotional activities and medical insurance strategies in UAE. Inability of patients to purchase costly medications significantly contributes to the problem of noncompliance and prescribing generic drugs should be advocated among prescribers unless there is an issue of bioavailability.

None of the prescriptions by GPS included injections whereas $8 \%$ of those issued by consultants contained injections. This may increase the possibility of non-compliance in case of expensive injectable formulations that cannot be afforded by the patient. Analysis of the number of drugs per encounter demonstrated that the incidence of poly-pharmacy is also higher in prescriptions issued by consultants than those by GPS. Such trend increases the risk of adverse effects and drug interactions. The later was evident in about $4 \%$ of encounters by both groups of prescribers. In general, one would expect a better prescribing pattern by consultants. Unfortunately, in the present study, this was not always the case. Focus in programs of medical continuing education on prescription writing may help improving the patterns of prescribing of both groups of prescribers.

The most commonly prescribed drugs were NSAIDs followed by antibiotics. The latter were reasonably prescribed by consultants (27\%). This is similar to our earlier findings in a hospital in Dubai [1]. On the other hand, GPs prescribed antibiotics in more than $40 \%$ of prescriptions. This is still far less than that prescribed in India [15]. The third common class was gastrointestinal drugs for consultants and multivitamins for GPs. No other medications were included in the prescriptions with multivitamins and the extensive use of multivitamins may be explained by the insistence of patients on receiving drugs to feel better.

Despite the fact that in some aspects of prescribing a more appropriate trend was shown by GPs than by consultants, prescribing by both groups still not ideal. Periodical monitoring of prescriptions can always be beneficial as it can pinpoint areas for improvement towards rational drug prescribing.

\section{Limitations of the Study}

The time constraint and difficulty in obtaining prescriptions did not allow us to analyze samples of prescriptions issued by consultants and GPs in other Emirates, therefore the results cannot be generalized to other Emirates than Dubai. Future studies will focus on prescriptions collected from various Emirates and hospitals.

\section{Conclusion}

A prescription should be wisely and appropriately written to include all the information that would be of help to the pharmacist who dispenses the medications and the patient who uses them. Periodical monitoring of prescriptions is useful as it can identify areas for improvement in prescription writing. Moreover, we suggest that interventions to improve prescribing behavior may include among others, feedback of results to the prescribers through personal interviews and incorporation of principles of rational prescription writing in medical curriculum and continuing medical education programs for both GPs and consultants.

\section{References}

[1] Sharif, S.I., Al-Shaqra, M., Hajjar, H., Shamout, A. and Wess, L. (2008) Patterns of Drug Prescribing in a Hospital in Dubai, United Arab Emirates. Libyan Journal of Medicine, 3, 10-12. http://dx.doi.org/10.4176/070928

[2] Irshaid, Y.M., Al Homrany, M., Hamdi, A.A., Adjepon-Yamoah, K.K. and Mahfouz, A.A. (2005) Compliance with Good Practice in Prescription Writing at Outpatient Clinic in Saudi Arabia. Eastern Mediterranean Health Journal, 11, 922-928.

[3] Meyer, T.A. (2000) Improving the Quality of the Order-Writing Process for Inpatient Orders and Outpatient Prescriptions. American Journal of Health-System Pharmacy, 57, S18-S12.

[4] Montastruc, F., Gardette, V., Cantet, C., Piau, A., Lapeyre-Mestre, M., Vellas, B., Montastruc, J.-L. and Andrieu, S. 
(2013) Potentially Inappropriate Medication Use among Patients with Alzheimer Disease in the REAL.FR Cohort: Be Aware of Atropinic and Benzodiazepine Drugs! European Journal of Clinical Pharmacology, 69, 1589-1597. http://dx.doi.org/10.1007/s00228-013-1506-8

[5] DeVries, T.P., et al., Eds. (1995) Guide to Good Prescribing: A Practical Manual. World Health Organization, Geneva, 51-5 (WHO/DAP/94.11).

[6] (1983) The Pharmaceutical Professions and Institutions. UAE Federal Law No: 4.

[7] Brahams, D. (1984) Legal Liability and the Negligent Prescription. Practitioner, 228, 444-445.

[8] Yousif, E., Ahmed, A.M., Abdalla, M.E. and Abdelgadir, M.A. (2006) Deficiencies in Medical Prescriptions in a Sudanese Hospital. Eastern Mediterranean Health Journal, 12, 915-918.

[9] WHO (1995) How to Investigate Drug Use in Health Facilities: Selected Drug Use Indicators. World Health Organization, Geneva, WHO/DAP/93.1.1995.

[10] Balbaid, O.M. and Al-Dawood, K.M. (1988) Assessment of Physician’s Prescribing Practices at Ministry of Health Hospitals in Jeddah City, Saudi Arabia. Saudi Medical Journal, 19, 28-35.

[11] Bawazir, S.A. (1992) Prescribing Patterns at Community Pharmacies in Saudi Arabia. International Pharmacy Journal, 6, 222-224.

[12] Sharif, S.I., Alabdouli, A.H. and Sharif, R.S. (2013) Drug Prescribing Trends in a General Hospital in Sharjah-United Arab Emirates. American Journal of Pharmacological Sciences, 1, 6-9. http://dx.doi.org/10.12691/ajps-1-1-2

[13] Velo, G.P. and Minuz, P. (2009) Medication Errors: Prescribing Faults and Prescription Errors. British Journal of Clinical Pharmacology, 67, 624-628. http://dx.doi.org/10.1111/j.1365-2125.2009.03425.x

[14] Bapna, J.S., Tekur, U., Gitanjali, B., Shashindran, C.H., Pradhan, S.C., Thulasimani, M., et al. (1992) Drug Utilization at Primary Health Care Level in Southern India. European Journal of Clinical Pharmacology, 43, 413-415. http://dx.doi.org/10.1007/BF02220618

[15] Kshirsagar, M.J., Langade, D., Patil, S. and Patki, P.S. (1998) Prescribing Patterns among Medical Practitioners in Pune. Bulletin World Health Organization, India. 